\title{
Good short-term survival rates for posterior resin composite restorations
}

\section{Abstracted from}

\author{
Ástvaldsdóttir Á, Dagerhamn J, van Dijken JW, Naimi-Akbar A, Sandborgh-Englund G, Tranæeus S, Nilsson M. \\ Longevity of posterior resin composite restorations in adults - A systematic review. J Dent 2015; 43: 934-954. \\ Address for correspondence: Á Ástvaldsdóttir, Division of Cariology, Department of Dental Medicine, \\ Karolinska Institutet, Huddinge, Sweden. E-mail: Alfheidur.Astvaldsdottir@ki.se
}

\section{Question: What is the longevity of posterior composite restorations in adults?}

Data sources PubMed, the Cochrane Library and the databases of the Centre for Reviews and Dissemination were searched Study selection Prospective randomised controlled trials (RCTs), clinical controlled trials (CCTs) and observational studies of Class I and/or Class II resin composite restorations with a sample size of more than 40 individuals or teeth and longer than four years follow-up were considered.

Results Eight studies involving a total of 910 restorations in 420 patients were included. Seven of the eight studies were completed by the same research group with follow-up times ranging from four to 12 years. There were 80 failures of restorations in total, ranging from two to 17 per study. A majority of the fractures of the restoration or the tooth and endodontic complications occurred during the first three years of follow-up. Caries occurred later, more than $75 \%$ after three years in service. The overall incidence rate for all causes of failure was 1.55 lost restorations per 100 restoration years. Survival rate at four years $=0.93(95 \% \mathrm{Cl} ; 0.91-0.95)$. One study (74 teeth) provided data at 12 years with a survival rate $=0.86(95 \% \mathrm{Cl} ; 0.82 ; 0.89)$. The most common biological reason for failure (a total of 31 restorations) was secondary caries, with or without fracture of the restoration. The overall quality of the evidence was low.

Conclusions The overall survival proportion of posterior resin composite restorations is high. The major reasons for failure are secondary caries and restoration fracture, which supports the importance of adequate follow-up time as secondary caries often occurred after three years or later.

\section{Commentary}

Following the international agreement to phase down the use of dental amalgam at the Minimata convention, ${ }^{1}$ the need for highpowered studies on the longevity of posterior alternatives has grown. Techniques based on the removal of healthy tooth tissue to accommodate the requirements of dental amalgam are out-dated. The next generation of dentists will be far more familiar with the placement of posterior composite resin restorations to conservatively manage carious lesions. ${ }^{2}$ These materials have continued to evolve so that they now have similar flexural strength, fracture toughness and tensile strength to dental amalgam. ${ }^{3}$ Despite the growing advantages and use of composite resin, studies to support its use posteriorly are still welcome.

The authors of this systematic review have aimed to determine the long-term survival of class I and/or II composite resin restorations in adults using prospective clinical trials of at least four years duration. The exclusion of retrospective studies may have reduced the risk of selection and observation bias in the overall survival analysis. Despite limiting their search to studies published after 1990, there has been a vast improvement in the mechanical properties of composite resin and adhesive techniques since then. A fairly comprehensive search strategy using a combination of free text and MeSH terms without language restrictions were utilised although only four electronic databases were searched. Authors and manufacturers were not contacted regarding any ongoing or unpublished research and the bibliographies of selected articles were not searched for further studies.

Five reviewers assessed abstracts. The full text articles of abstracts fulfilling the inclusion criteria were evaluated for risk of bias using the Swedish Council on Health Technology Assessment (SBU) standardised checklists. Studies with a moderate to low risk of bias were assessed for overall quality using the GRADE criteria. At each stage, reviewers who were authors or co-authors of a paper were excluded from the evaluation process. Data from eight studies published between 2005 and 2013 were included in the overall analysis although seven studies originated from the same research group. A co-author of the review was involved in seven of the included studies. This should be given due consideration when reflecting on the external validity of this study. Of the included studies, only two were graded as high quality. The remaining six were of moderate quality.

A total of 910 restorations in 420 patients over a follow-up period of four to 12 years were analysed. All studies employed the intra-individual comparison and modified USPHS criteria for 
evaluating the restorations. Biological failures (secondary caries = 31 , tooth fracture $=15$, endodontic complications $=7$ ) accounted for the majority of failures, reinforcing the findings of previous reviews suggesting that caries and fractures are the most common mechanisms of failure. ${ }^{4,5}$ Technical failures (27) made up the remainder. The total follow-up at risk for failure was 62,030 months and the overall incidence rate for all causes of failure was 1.55 lost restorations per 100 restoration years. Four-year survival was high at 0.93 but the overall quality of evidence was regarded as low. Only one study reported survival after 12 years (0.86) but the quality of this particular study was even lower. The failure rate for single surface restorations was lower than multi-surface restorations supporting the conclusions of a recent systematic review. ${ }^{4}$

Of the included studies, seven did not use rubber dam and only one reported the use of a fourth generation three-step bonding system. Despite this, the authors conclude that the findings of this systematic review cannot be applied to an ordinary clinical or 'effectiveness' setting as the included studies were, in their opinion, conducted under optimal conditions. They may be alluding to the likelihood of increased motivation in the cohort of patients undergoing treatment within a hospital setting. Nevertheless, differences in the type of composite, method of application and adhesive systems within and between studies may limit the overall applicability of this review. Six studies reported the percentage of patients with a high susceptibility to caries ( mean $=32.5 \%$ ). Previous studies have demonstrated an increased risk of failure in this group of patients, ${ }^{4,5,6}$ highlighting the importance of a preventive and conservative approach to caries management.

A recent Cochrane systematic review has shown that composite resin restorations have a higher risk of failure and increased risk of secondary caries when compared to amalgam restorations. ${ }^{7}$ The looming phase-down of amalgam will mean that the use of composite will inevitably increase. As it stands, there is still insufficient longterm evidence to show composite outperforming amalgam. This review reports a high rate of survival over a short period of time without any comparison to amalgam. The authors acknowledge that studies reporting the survival of posterior composite resin restorations of longer duration are required. Conversely, the outcomes from these studies may prove obsolete due to the rapid advances in adhesive dentistry.

\section{Practice points}

- The appropriate handling and placement of posterior composite resin restorations in patients with low caries risk can lead to higher rates of survival

- Patients must appreciate the key role they play in the longevity of their restorations through good oral hygiene measures and dietary habits.

Ravi Chauhan

Restorative Dentistry, King's College Dental Hospital and William Harvey Hospital (Kent, Surrey and Sussex Deanery), UK

1. United Nations Environment Programme. Minamata convention agreed by nations. UNEP, 2013. Online article available at http://www.unep.org/newscentre/default.asp $x$ ?DocumentID=2702\&ArticlelD=9373 [Accessed November 2015].

2. Lynch $C D$, Frazier KB, McConnell RJ, Blum IR, Wilson NH. State-of-the-art techniques in operative dentistry: contemporary teaching of posterior composites in UK and Irish dental schools. Br Dent / 2010; 209: 129-136.

3. Ferracane JL. Resin composite - state of the art. Dent Mater 2011; 27: 29-38.

4. Opdam NJ, van de Sande FH, Bronkhorst E, et al. Longevity of posterior composite restorations: a systematic review and meta-analysis. J Dent Res 2014; 93: 943-949.

5. Demarco FF, Corrêa MB, Cenci MS, Moraes RR, Opdam NJ. Longevity of posterior composite restorations: not only a matter of materials. Dent Mater 2012; 28: 87-101.

6. Opdam NJ, Bronkhorst EM, Loomans BA, Huysmans MC. 12-year survival of composite vs. amalgam restorations. J Dent Res 2010; 89: 1063-1067.

7. Rasines Alcaraz MG, Vietz-Keenan A, Sahrmann P, Schmidlin PR, Davis D, IheozorEjiofor Z. Direct composite resin fillings versus amalgam fillings for permanent or adult posterior teeth. Cochrane Database Syst Rev 2014; 3: CD005620.

Evidence-Based Dentistry (2015) 16, 114-115. doi:10.1038/sj.ebd.6401135 